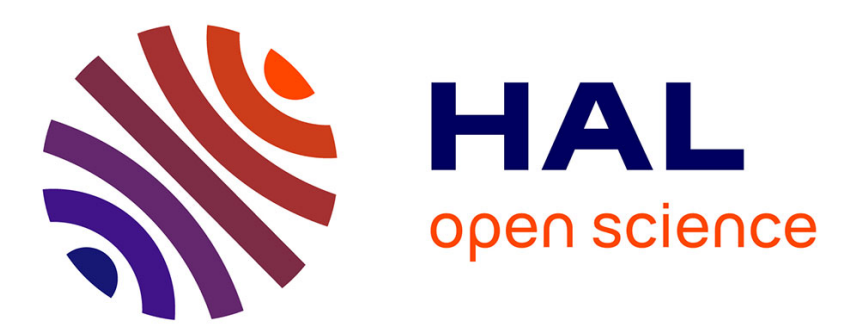

\title{
Light-induced retinal damage using different light sources, protocols and rat strains reveals LED phototoxicity
}

A. Krigel, M. Berdugo, E. Picard, R. Levy-Boukris, I. Jaadane, L. Jonet, M. Dernigoghossian, C. Andrieu-Soler, Alicia Torriglia, F. Behar-Cohen

\section{To cite this version:}

A. Krigel, M. Berdugo, E. Picard, R. Levy-Boukris, I. Jaadane, et al.. Light-induced retinal damage using different light sources, protocols and rat strains reveals LED phototoxicity. Neuroscience, 2016, 10.1016/j.neuroscience.2016.10.015 . hal-01383394

\section{HAL Id: hal-01383394 https://hal.sorbonne-universite.fr/hal-01383394}

Submitted on 18 Oct 2016

HAL is a multi-disciplinary open access archive for the deposit and dissemination of scientific research documents, whether they are published or not. The documents may come from teaching and research institutions in France or abroad, or from public or private research centers.
L'archive ouverte pluridisciplinaire HAL, est destinée au dépôt et à la diffusion de documents scientifiques de niveau recherche, publiés ou non, émanant des établissements d'enseignement et de recherche français ou étrangers, des laboratoires publics ou privés. 
Light-induced retinal damage using different light sources, protocols and rat strains reveals LED phototoxicity.

Krigel A, Berdugo M, Picard E, Levy-Boukris R, Jaadane I, Jonet L, Dernigoghossian M, , Andrieu-Soler C, Torriglia A, Behar-Cohen F

INSERM U1138. Centre de Recherches des Cordeliers. Université Paris Descartes, Université Pierre et Marie Curie. Paris. France

Corresponding authors:

Alicia Torriglia: alicia.torriglia@inserm.fr

Francine Behar-Cohen: francine.behar@gmail.com 


\section{Highlights}

- White light from LED induces a stronger retinal degeneration in albino rats than light from fluorocompact lamps when used at the same luminance.

- Pigmentation induces very little protection in LED exposed rats at high luminance.

- Exposure to LED induces breakdown of the external blood-retinal barrier.

\section{Abbreviations:}

AMD: Age related macular degeneration.

BRB: Blood retinal Barrier.

CCFL: Cold Cathode fluorescent lamps

CFL: fluorocompact lamp

ERG: electro retinogramme

INL: Inner nuclear layer

LE: Long Evans

LED: Light Emitting diode

ONL: outer nuclear layer

PNA: peanut agglutinine

W: Wistar

Authors' involvement: AK made most of the experiments, MB performed the ERG, $D M$ and IJ made some of the immunostaining experiments, $L J$ and $R L$ gave technical assistance, CAS and FBC conceived the experiments. AK, MB,EP, AT and FBC wrote the paper. 


\section{Abstract}

To save energy, the European directives from the Eco-design of Energy Using Products (2005/32/CE) has recommended the replacement of incandescent lamps by more economic devices such as Light Emitting Diodes (LEDs). However, the emission spectrum of these devices is enriched in blue radiations, known to be potentially dangerous to the retina. Recent studies showed that light exposure contribute to the onset of early stages of Age related macular degeneration (AMD). Here, we investigate, in albinos and pigmented rats, the effects of different exposure protocols. Twenty-four hours exposure at high luminance was compared to a cyclic (dark/light) exposure at domestic levels for 1 week and 1 month, using different LEDs (Cold-white, blue and green), as well as fluorocompacts bulbs and fluorescent tubes. The data suggest that the blue component of the white-LED may cause retinal toxicity at occupational domestic illuminance and not only in extreme experimental conditions, as previously reported. It is important to note that the current regulations and standards have been established on the basis of acute light exposure and do not take into account the effects of repeated exposure.

Key words: retina, light emitting diodes, phototoxicity, pigmented rats, chronic light exposure. 


\section{Introduction}

Artificial light consumes near to $20 \%$ of the world electricity production. To save energy, the European directives from the Eco-design of Energy Using Products (2005/32/CE) have recommended the replacement of incandescent lamps by more economic devices such as Light Emitting Diodes (LED). By 2019, LED will be the major domestic and public light source. LEDs emit mono chromic lights, and the less expensive and currently used method to produce white light from LED is to combine a blue LED with a yellow phosphore coverage. The resulting spectrum is enriched in blue radiations, known to be potentially dangerous to the retina (Algvere et al., 2006). The other concerns are the high luminance level and the visual discomfort due to the punctual character of the emitting surfaces.

The role of sunlight exposure in the development and/or aggravation of retinal diseases and particularly Age-Related Macular Degeneration (AMD), which is associated with oxidative stress and inflammation, has been disputed for years (Ardeljan and Chan, 2013, Pinazo-Duran et al., 2014, McHarg et al., 2015). Indeed, cumulative light exposure, and particularly retinal exposure is difficult to estimate (Sliney, 2005). However, recently, based on large population studies, light exposure has been clearly recognized as a contributing factor in the appearance of the early stages of AMD (Klein et al., 2007, Sui et al., 2013).

In this context, light exposure must be considered as part of the environmental factors that can influence multiple physiologic processes and potentially impact pathologic retinal aging. The massive conversion from incandescent lights to LED incorporating devices in domestic lighting should be examined in more depth as recommended by the governmental agencies (ANSES report,(Saisine 2008SA0408) French Agency for Food, Environmental and Occupational Health and Safety).

Risk evaluation is based on epidemiologic studies, experimental results and exposure scenarios. But, whilst extreme acute exposures to high luminance lighting systems are frequently used in various models of light-induced retinal degeneration, few studies have evaluated the effects of different light sources in conditions close to domestic use (Peng et al., 2012, Shang et al., 2014).

In this study, we investigate, in albinos and pigmented rats, the effects of 
different exposure protocols. Twenty-four hours exposure at high luminance was compared to a chronic cyclic (dark/light) exposure at domestic levels for 1 week and 1 month, using different LEDs (Cold-white, blue and green), as well as fluorocompacts bulbs (CFL) and Cold Cathode fluorescent lamps (CCFL) (fluorescent tubes). 


\section{Materials and Methods}

\section{Animals}

8 weeks-old albino Wistar (W) and pigmented Long Evans (LE) rats (Janvier laboratory, Le Genest St Isle, France) were used in these experiments. At least 4 rats were used per exposure condition and per time point. Rats were maintained on a 12 $\mathrm{h} / 12 \mathrm{~h}$ light-dark (LD) cycle at $22{ }^{\circ} \mathrm{C}$ at a luminance below 250 lux, for 21 days before light-exposure experiments. All experimental procedures were performed in accordance with the Association for Research in Vision and Ophthalmology (ARVO) statement for the use of animals in Ophthalmic and Vision Research. Experimental procedures were submitted and approved by the local ethics committee European Council Charles Darwin, University Paris Descartes (Authorization $\mathrm{N}^{\circ}-05$, Ce5/2012/019, A75-580).

\section{Light sources}

We used 2 types of lighting devices. For exposure to white LED, commercial cold white LED panel generating 2,300 lumens during 24 hours was used. The LED panel was placed above 8 transparent cages, placed on white surfaces, leaving enough space for air circulation and constant temperature maintenance at $21^{\circ} \mathrm{C}$. The illuminance measured at the rats' eyes position was 6000 lux (Photometre DT-8809A, CEM, China).

For long-term exposure, specific devices were built and characterized by Statice, France (Figure 1A). Metallic boxes contained rows of LED with a diffuser in order to improve the directional uniformity of the radiation and avoid punctate sources. Alternatively, CCFL or CFL were uniformly distributed around the metal cages. Each cage was placed in a metallic device that was then placed in a ventilated cupboard allowing for a constant $21^{\circ} \mathrm{C}$ temperature control (Figure $1 \mathrm{~A}$ ). The light intensity was controllable and the distribution of light in the cage was homogenous whatever the rat position. Different types of LEDs were used: cold-white LED (pure white 6300K), blue LED (royal blue 455-465nm), and green LED (520$535 \mathrm{~nm})(Z$-power LED, Seoul Semiconductor, Korea). Exposure intensity was spectrophotometrically measured by Statice. 


\section{Exposure protocols}

Acute exposure: LE and W rats were maintained in a cyclic light/dark (250lux, $12 \mathrm{~h} / 12 \mathrm{~h}$ ) environment for 21 days. The day before light exposure, rats were darkadapted for 16 hours. The next day, pupils were dilated with 1\% atropine (Alcon, Norvartis, Rueil Malmaison, France) under dim light, and rats were isolated in separate cages containing enough food for one day. After 24 hours of exposure, rats were placed again in a cyclic light/dark (250lux, 12h/12h) environment for 7 days and sacrificed for histology and immunofluorescence analysis. Control rats were submitted to the same pre conditioning protocol but not exposed to light. Different types of light sources and light intensities were used as detailed in Figure 1B. For cold-white LED, different light intensities were tested from 6000 lux, to 1500, 1000 and 500 lux. Blue and green LEDs were used at 500 lux which is the domestic classic light intensity. CFL was used at 6000 lux and 500 lux, CCFL at 6000 lux. Illuminance was measured at the level of the rat eye.

Long-term exposures: Rats (LE and W) were maintained in a cyclic light/dark (250lux, $12 \mathrm{~h} / 12 \mathrm{~h}$ ) environment for 21 days, then placed in specific cages for chronic cyclic exposure to different types of light at 500 lux: CFL, white, green and blue LEDs. Animals were sacrificed right after 8 or 28 days of exposure. For the long-term protocol and in order to be as close to domestic light as possible, rat pupils were not dilated.

\section{Histology and photoreceptors quantification}

Animals were sacrificed with sodium pentobarbital (> $60 \mathrm{mg} / \mathrm{kg}$, intraperitoneal) and eyes were enucleated. Eyes were oriented (superior/ inferior pole), rinsed in cold PBS for $1 \mathrm{~h}$, transferred to an ascending series of ethanol solutions $(70 \%, 96 \%$, for 2 h) then put in 2 successive bathes of infiltration resin and ethanol (1:1). Finally, they were embedded in the same resin with catalyzer. $5 \mu \mathrm{m}$ thick sections were placed at 37 degrees for $24 \mathrm{~h}$ and then stained with toluidine blue and incubated at 37 degrees for 24 hours before microscope observation and photography.

Photoreceptor quantification was done by counting for each retinal section the number of nuclei in the Outer Nuclear Layer (ONL) from the optic nerve each $0,5 \mathrm{~mm}$ $(0.5,1,1.5,2,2.5,3,3.5$ and $4 \mathrm{~mm})$ in both directions (superior and inferior retina). Mosaic pictures were done for masked counting. 


\section{Immunofluorescence and TUNEL assay}

Freshly enucleated eyes ( $n=3-4$ per time point)(superior pole tagged with suture) were fixed for 2 hours with 4\% paraformaldehyde (PAF, Inland Europe, Conflans sur Lanterne, Fr) in 1X phosphate-buffered saline (PBS, Gibco distributed by Life Technologies), washed with PBS, infiltrated with increased concentrations of sucrose and then, mounted in Tissue Tek O.C.T. (Siemens Medical, Puteaux, Fr).

Immunofluorescence was performed on $10 \mu \mathrm{m}$-thick sections. Cryosections were incubated with different primary antibodies: Rabbit anti GFAP (Dako Agilent Tech, USA), rabbit anti-lba1 (Cell Signaling, Biorad, France); mouse monoclonal anti-CD68 (ED1) (Santa Cruz, USA), mouse monoclonal anti Rho4D2 (Abcam). Rods and cones were respectively labeled with anti-rhodopsin (Rho4D2, R.S. Molday) and peanut agglutinin conjugated with fluorescein isothiocyanate (Sigma). Control sections were incubated with rabbit non-immune serum (Invitrogen, Cergy Pontoise, Fr) or without primary antibodies. The corresponding Alexa-conjugated secondary antibodies (Invitrogen) were used to reveal the primary antibodies. Sections were counterstained with 4.6-diamidino-2-phenylindole (DAPI, Sigma). TUNEL assay was performed on all sections following the manufacturer's instructions (Roche Diagnostics, Mannheim, Germany).

The sections were viewed with a fluorescence microscope (BX51, Olympus, Rungis, Fr) or confocal microscope (LSM 510 laser scanning microscope Zeiss, Carl Zeiss, Le Pecq, Fr) and photographed using identical exposure parameters for all samples to be compared.

\section{Electroretinograms (ERG)}

Full-field ERG responses were recorded before and after the end of light exposure. Rats were dark-adapted for 18 hours and anesthetized by an intramuscular injection of a mixture of ketamine and xylazine. The cornea was desensitized with a drop of oxybuprocaine (Novesine@ Novartis Ophthalmics, Basel, Switzerland) and the pupils were dilated with a drop of tropicamide (Tropicamide@), Novartis Ophthalmics). Gold wire ring electrodes were placed on the corneas of both eyes and stainless steel needle electrodes inserted into the forehead served as references electrodes. A needle electrode subcutaneously inserted at the base of the animal tail was used for 
grounding. All these manipulations were performed under dim red light. Measurements were performed using the commercial Ganzfeld VisioSystem device (Siem Biomedicale, Nîmes, Fr). For scotopic electroretinograms in the dark-adapted state, flash intensities ranged from 0.0003 to $10 \mathrm{~cd} . \mathrm{s} / \mathrm{m}^{2}$. Five flashes of $10 \mathrm{~ms}$ per intensity were applied at a frequency of $0.5 \mathrm{~Hz}$. for -30 to $0 \mathrm{~dB}$ and for $30 \mathrm{~ms}$ for $10 \mathrm{~cd} . \mathrm{s} / \mathrm{m} 2(0 \mathrm{~dB})$. Five responses were averaged. Amplitudes of a-waves (negative waves) were measured from the baseline to the bottom of the a-wave, b-wave amplitudes (positive waves) were measured from the bottom of the a-wave trough to the peak of the b-wave. Implicit times of the $a$ - and $b$ - waves were measured from time of stimulus to peaks. Results were expressed in microvolts $(\mu \mathrm{V})$ for amplitudes and milliseconds (ms) for implicit times. The data obtained from each eye belonging to the same experimental group were averaged. We analyzed the variation of each parameter of the ERG before-after light exposure. The a-wave is a negative wave, thus a positive variation is an alteration of the a-wave amplitude. The b-wave is a positive wave, thus a negative variation is an alteration of the b-wave amplitude. When the variation of the implicit time is positive, it also means an alteration of the function.

\section{Statistics}

Data are presented as the mean \pm SD. Data were evaluated using R-cran software. Kruskal-Wallis test was done to test for normality $(p=0.05)$, then a post hoc analysis was performed. For a one to one comparison a Mann-Whitney test was done and for a multiple comparison, a Dunn test and a Conover Imann test were performed. $\mathrm{P}<$ 0.05 was considered as significant. 


\section{Results}

\section{All light sources induced photoreceptor damage in both pigmented and albino rats after acute exposure at $\mathbf{6 0 0 0}$ lux with dilated pupil}

The aim of this first protocol was: 1) to compare light sensitivity of pigmented and albino rats to high and low light intensities, 2) to compare different light sources at high and low intensities 3) to determine the toxic threshold level of LED.

After 24 hrs of light exposure at 6000 lux, a clinical difference was observed between rats exposed to white LEDs as compared to rats exposed to other light sources. In LED exposed rats an important edema of the eyelids and the conjunctiva, as well as the face of the animals was observed (not shown).

Under these conditions a significant loss of photoreceptors was observed in the superior retina of both LE and $\mathrm{W}$ rats with all types of light sources (Figure $2 \mathrm{~A}$ and $2 \mathrm{~B}$ ). At this light intensity, loss of photoreceptors cells was also observed at a lesser extent in the inferior part of the retina. The loss of cells was significantly less in pigmented (LE) rats as compared to albino (W) rats (not shown). In LE rats, white LED, CCFL and CFL induce similar loss of photoreceptors in the superior retina (Figure 2A), but in W rats, the most important loss of cells was induced by CCFL (fluorescent tube) (Figure 2B). Interestingly, W rats seem less sensitive to CFL than to other devices and less sensitive to CFL than LE rats (Fig 2B).

Immunohistochemistry allowed a more detailed analysis of the retinal damage caused by LED exposure (Figure 3). In LE rats, macroglial activation was more intense than in $W$ rats with sub retinal glial Müller cell migration (Figure 3 inset). In W rats, macroglia was less activated but numerous GFAP dendritiform cells were localized in the outer plexiform layer (OPL) and in the sub retinal space (Figure 3, W-LED asterisks. In both strains, rods (Rho 4D2) and cones (PNA) were severely damaged but whilst some rods still remained, no cones were left (Figure 3 Rho4D2 and PNA)). IBA1/ ED1-co labeling indicated that an intense inflammatory reaction was present in $\mathrm{W}$ illuminated rats with numerous IBA1 positive cells in the inner retina and IBA1/ ED1 co-labeled activated cells in the sub retinal space. In LE rats, the inflammatory reaction was mostly confined to the outer retina where activated round IBA1 positive microglial cells, ED1 positive macrophages and co labeled cells were observed.

Concerning the other lighting devices, immunohistochemistry showed intense activation of glial Müller cells exposed to CCFL or CFL in both pigmented and non- 
pigmented rats (Figure 4). The most damaged outer retina with the most intense sub retinal gliosis was seen in Wistar rats exposed to CCFL. With both CCFL and CFL, both rods and cones were altered but with a more intense loss of cones as shown by PNA labeling. An intense inflammatory reaction involving both microglial cells and macrophages was observed in all retinas with a higher infiltration of ED1 positive/ IBA1 negative cells, suggesting infiltrating macrophages, in LE rats as compared to albino rats, where the microglial activation was intense (Figure 4 insets).

\section{At 500 lux, cold-white LED, but not CFL induced photoreceptor damage both in albino and pigmented rats after acute exposure and dilated pupil}

The results presented above suggested that pigmented rats were not completely protected from retinal degeneration induced by LEDs as compared to their albino counterpart. We tested then decreasing luminances to detect different sensitivities to light toxicity between the pigmented and the albino strains. Using the same acute protocol (figure 2B), the effects of LED light at 500, 1000 and 1500 lux were evaluated on LE and $W$ rats. At 500 lux, which is the recommended light intensity for domestic lighting, CFL did not induce any photoreceptor cells loss, neither in pigmented nor in albino rats at the inferior retina level (Figure 5, left column). At the superior retina, however, the same dose induced a decrease in the number of photoreceptor's rows when LEDs light was used. With this light source, a dose-dependent loss of photoreceptor cells was observed after acute illumination of both albino $\mathrm{W}$ and pigmented LE rats with dilated pupils (Figure 5, right column). Only at 1500 lux, inferior retinas of both $W$ and LE were affected with significantly more photoreceptors loss in W as compared LE (Figure 5, left column). These results clearly show that at the same light intensity, different light sources do not exert the same toxicity: LED are more toxic for the retina than CFL. Importantly, in these experiments, pigmented rats also showed light damage at domestic used intensity.

Analysis of the inflammatory reaction by immunofluorescence showed that a LED exposure for $24 \mathrm{hrs}$ at 500 lux, resulted in an activated macroglia in the superior retina of both LE and $\mathrm{W}$ rats as shown by GFAP staining (Figure 6). In LE rats, GFAP positive macroglial cells were observed migrating in the outer retina (Figure 6, inset). Although the decrease in photoreceptors nuclei was higher in $W$ than in LE rats, in LE rats, cones labeled by PNA have been completely lost although some remained in W rats (Figure 6). The decrease in rod labeling (Rho4D2) followed the same pattern in both types of 
animals.

At 1500 lux (Figure 6 right), a more intense damage was observed in both $\mathrm{W}$ and LE rats, where a major macroglial activation associated to a complete loss of cones and rods was also observed. Note that although ONL thickness was decreased in W exposed rats, the total retinal thickness was not decreased due to retinal edema.

\section{The retinal pigment epithelium is also involved.}

The presence of retinal edema suggested that the blood retinal barrier (BRB) could be damaged. The outer BRB is formed by the tight-junction retinal pigment epithelium that in physiologic conditions do not allow the passage of albumin from the choroid to the retina (Rizzolo, 1997). Breakdown of the retinal pigment epithelial barrier was evaluated by albumin labeling using an anti-rat serum albumin. After $24 \mathrm{~h}$ of LED exposure we saw the presence of albumin in the superior retina of both pigmented and albino rats (Figure 7); some leakage was also seen, in a lesser extent in the inferior retina of albino rats.

\section{Long-term exposure to LED at 500 lux, in cyclic (light/dark) conditions induced retinal damage only in albino rats but not in pigmented rats.}

Tissues modifications

Next we investigated the retinal effect of different light sources (CFL and LED) and colors (white, blue and green LEDs) after 1 week and 1 month of cyclic exposure at 500 lux without pupil dilation (mimicking long-term domestic lighting). After 1 week of exposure, retinal damage was different in $\mathrm{W}$ albinos and LE pigmented rats. In $\mathrm{W}$ rats, retinal cell loss following 1 week of exposure was observed only in the superior retina of rats exposed to blue-LEDs (Figure $8 \mathrm{~A}$ and 9A). After 1 month of exposure, all LEDs induced retinal damage in the superior retina, and only blue and green LEDs induced damage also in the inferior retina (Figure $8 \mathrm{~B}$ ). In contrast, LE pigmented rats did not present any significant retinal cell loss under these conditions (Figure 9 A and B) (the number of photoreceptors' nuclei were compared to rats exposed to CFL that had no effect on photorecpetors' number, see Figure 3). The immunohistochemical analysis of these retinas, exposed to 1month cyclic illumination, showed a conserved Rho4D2 labeling in LE rats, a decrease of photoreceptors's outer segment in W rats that include a loss or an alteration of cones when exposed to blue or green lights (Figure 10). Interestingly, the results shown on figure 9 and the rhodopsin and cones labeling in figure 10 suggested that, using the present protocol, we did not induce any damage to the retina of pigmented rats. However, when 
analyzing the expression of GFAP it appeared that exposure to green LED did not change the expression of this protein as compared to the control (see Figure 3), whilst exposure to white LEDs and blue LEDs induced the expression of this protein even in LE rats.

Functional modifications

In $W$ and LE rats, we recorded the full-field electroretinograms (ERG, visual function) of both eyes before and after a 1-month of long cyclic illumination to white LEDs at 500 lux, We analyzed, for each ERG parameter, the variation before and after light exposure, noted "delta". As the a-wave is a negative wave, a positive variation represents a decrease of the $a$-wave amplitude. As the b-wave is a positive wave, a negative variation of the $b$-wave amplitude. We showed that both scotopic a- and b- waves' amplitudes are impaired by white LED illumination, in albino (W) as well as in pigmented (LE) rats (Figure 11). In LE, the a-wave (photoreceptors function) deterioration occurred with a little delay when compared to W rats, suggesting that W photoreceptors, were more sensitive than LE's. Whereas, LE b-wave (inner retina function) is slightly less deteriorated than W rats's bwave (non significant trend, however). Implicit times of both a- and b-waves are not modified by this illumination protocol, neither in W nor in LE rats.

It is interesting to note that, following this 1-month long cyclic illumination protocol, ONL thickness was slightly reduced only in the $\mathrm{W}$ superior retina, while it was unaltered in $\mathrm{W}$ inferior and LE superior and inferior retinas; whereas, at the same time, macroglia was already activated, and ERG a- and b- waves were already impaired.

\section{Discussion}

The aim of this study was to compare different commercial light sources, available for domestic lighting on different animal strains, pigmented and non-pigmented rats and with different exposure scenarios. Different conditions of pupil dilation, as well as different exposure conditions were compared. Our purpose was to reproduce both the acute and extreme conditions used in light-induced retinal damage models and the domestic lighting conditions, which are the more representative of potential toxic effects for humans. Indeed, many studies have extensively analyzed the effect of acute exposure to high light intensity in order to decipher the mechanisms of light-induced retinal toxicity (Stone et al., 1999, Wenzel et al., 2005, Chahory et al., 2010, Organisciak and Vaughan, 2010), but much less experiments have been conducted to analyze the risks of new LED lighting systems in domestic lighting conditions (Shang et al., 2014, Jaadane et al., 2015). Recently, we 
performed an extended analysis of the mechanisms of LED-induced retinal cell toxicity on albino rats, showing that unexpectedly, not only apoptosis was induced but also necrotic cell death, particularly with blue LEDs (Jaadane et al., 2015). This necrotic death triggered an important inflammatory response as observed in our experiments, even at domestic light intensity on albino rats. The present study was not designed to study mechanisms but mostly to define the toxicity threshold conditions of different LEDs in occupational and domestic conditions.

As expected, at high illuminance, i.e. $6000 \mathrm{lux}$, with dilated pupils, retinal damage was observed equally with all light sources, CCFL, CFL and white LEDs, all induced a significant reduction in the photoreceptor layer thickness, intense macroglial reaction with sub retinal proliferation, rods segment fragmentation, loss of cones and intense microglial activation and macrophages infiltration at 8 days after light exposure. Inflammatory reaction seemed more diffuse all over the retina in LED exposed $\mathrm{W}$ rats as compared to LE rats but this was not specifically quantified. This could be related to the necrotic cell death observed when albino rats were exposed to blue-light containing LEDs (Jaadane et al., 2015) or to an enhanced inflammatory reactivity of this particular rat strain. More surprisingly, after $24 \mathrm{hrs}$ of continuous exposure of rats with dilated pupils, to white-cold LED at 500 lux, a significant reduction of ONL thickness was found not only in albinos but also, to a lesser extent in pigmented rats. Obviously, in physiologic conditions, when exposed to light, pupil constriction very efficiently reduces retinal exposure, protecting from toxicity (Sliney, 2005). This was confirmed by the absence of ONL reduction when pigmented rats were submitted to same lighting conditions but without dilation of the pupil (Figures 8 and 9). It is important to note that in dilated conditions, at the same illuminance, CFL did not cause any damage neither in the albino nor in the pigmented rat, demonstrating that different light sources do not exert the same potential retinal risk. Reduction of the photoreceptor layer was correlated to the illuminance produced by the white-cold LEDs demonstrating a dose-response toxic effect. The role of blue radiations are well recognized and have been also confirmed using blue LEDs in albino rats, where intense cone toxicity was shown at 200-lux illuminance measured on the rat cornea (OrtinMartinez et al., 2014). Here, blue LEDs at 500-lux illuminance were toxic after 24 hrs of continuous exposure even in pigmented not dilated pupil rats, which questions the potential effects of domestic blue light on human retinas, commonly used for decoration purposes.

Acute LED-induced damage were shown by several groups. In 2001, Dawson 
showed that the direct exposure of monkey retinas to a blue LED (460nm, corneal irradiance over $10 \mathrm{~J} / \mathrm{cm}^{2}$ ) induced macular lesions similar to those induced by an argon laser (458nm) (Dawson et al., 2001). Macular lesions were also observed in monkey by Ueda et al after direct exposure to a $465 \mathrm{~nm}$ LED (Ueda et al., 2009). More recently, Mukai et al. exposed monkey retina to LED contact lens for $8 \mathrm{hrs}$ at an illuminance of 7000 lux, observing both morphologic and functional changes on ERG and spectral domainOCT, that corresponded to intracellular vacuolization and irregularity of the lamellar structure of segments. Interestingly the ERG changes were transitory and returned to normal values 14 days after the experiments (Mukai et al., 2012).

Extrapolation of acute light exposure to mid and long term is therefore questionable and whether successive transitory damage may cause long term toxicity remains to be demonstrated.

Only one study was conducted to specifically answer the question of repeated LED exposure toxicity on albino rats. Albino rats were dark-adapted for 14 days and then submitted to 750-lux white LED cyclic exposure for 28 days. Under such conditions, severe retinal damage were observed associating necrotic and apoptotic cell death (Shang et al., 2014). Our experiments confirm these observations using cyclic exposure of young albino wistar rats to white LED, without pupil dilation and without extensive dark adaptation. To our knowledge, this is the first study comparing cyclic dark/light long-term exposure to white, blue and green LEDs, conducted also on pigmented rats without pupil dilation. Interestingly, as compared to albino rats, no significant morphological retinal damage was observed in pigmented animals under these lighting conditions. However, in addition to impaired retinal function, some other oxidative stress markers, like GFAP overexpression appear, suggesting that infra clinical oxidative stress, cumulated over years, could induce other types of retinal alterations, not examined in these experiments, and difficult to detect in a relevant animal experiment.

Many factors influence retinal exposure and retinal toxicity, including retinal pigment epithelium pigmentation, pupil diameter, geometry of the face and the nature of the light radiations, including its spectrum, its intensity, the exposure sequence and timing of exposure (Youssef et al., 2011, Hunter et al., 2012). Age, lens color (increasing yellow pigment with aging), stress-induced steroids, pre-existing retinal pathology also influence light sensitivity. Extrapolation of animal experiments are challenging and particularly, rats that do not have a macula and therefore do not recapitulate human retina characteristics. But, comparisons were made in this study in a very controlled manner which allow 
compare the effects of different light sources. It shows that at the same illuminance and under similar conditions, white, blue and green LEDs provoke retinal damage, while CFLs do not. They also highlight once more, the toxicity of blue light and particularly of blueLEDs.

Taken together these data suggest that the blue component of the white-LED may cause retinal toxicity at occupational domestic illuminance and not only in extreme experimental conditions, as previously suspected (Behar-Cohen et al., 2011, van Norren and Gorgels, 2011). It is important to note that the current regulations and standards have been established on the base of acute light exposure and do not take into account the effects of repeated exposure (Jarrett and Boulton, 2012, Protection, 2013). Moreover, no clear surrogate marker of light-induced retinal stress is used to detect sub-clinical retinal damage, that with time, could induce a different type of toxicity such as the one seen in AMD (Marquioni-Ramella and Suburo, 2015).

Since LEDs will very soon become the predominant light source in our domestic environment, it becomes urgent to establish a safe way to use them in the short and long term.

Acknowledgments: This work was supported by the region lle de France, grant to FBC and AK and by ADEME, Retinaled grant to IJ and AT.

\section{References}

Algvere PV, Marshall J, Seregard S (Age-related maculopathy and the impact of blue light hazard. Acta Ophthalmol Scand 84:4-15.2006).

Ardeljan D, Chan CC (Aging is not a disease: distinguishing age-related macular degeneration from aging. Prog Retin Eye Res 37:68-89.2013).

Behar-Cohen F, Martinsons C, Vienot F, Zissis G, Barlier-Salsi A, Cesarini JP, Enouf O, Garcia M, Picaud S, Attia D (Light-emitting diodes (LED) for domestic lighting: any risks for the eye? Prog Retin Eye Res 30:239-257.2011).

Chahory S, Keller N, Martin E, Omri B, Crisanti P, Torriglia A (Light induced retinal degeneration activates a caspase-independent pathway involving cathepsin $D$. Neurochem Int 57:278-287.2010).

Dawson W, Nakanishi-Ueda T, Armstrong D, Reitze D, Samuelson D, Hope M, Fukuda S, Matsuishi M, Ozawa T, Ueda T, Koide R (Local fundus response to blue (LED and 
laser) and infrared (LED and laser) sources. Exp Eye Res 73:137-147.2001).

Hunter JJ, Morgan JI, Merigan WH, Sliney DH, Sparrow JR, Williams DR (The susceptibility of the retina to photochemical damage from visible light. Prog Retin Eye Res 31:28-42.2012).

Jaadane I, Boulenguez P, Chahory S, Carre S, Savoldelli M, Jonet L, Behar-Cohen F, Martinsons C, Torriglia A (Retinal damage induced by commercial light emitting diodes (LEDs). Free Radic Biol Med 84:373-384.2015).

Jarrett SG, Boulton ME (Consequences of oxidative stress in age-related macular degeneration. Mol Aspects Med 33:399-417.2012).

Klein R, Klein BE, Knudtson MD, Meuer SM, Swift M, Gangnon RE (Fifteen-year cumulative incidence of age-related macular degeneration: the Beaver Dam Eye Study. Ophthalmology 114:253-262.2007).

Marquioni-Ramella MD, Suburo AM (Photo-damage, photo-protection and age-related macular degeneration. Photochem Photobiol Sci 14:1560-1577.2015).

McHarg S, Clark SJ, Day AJ, Bishop PN (Age-related macular degeneration and the role of the complement system. Mol Immunol 67:43-50.2015).

Mukai R, Akiyama H, Tajika Y, Shimoda Y, Yorifuji H, Kishi S (Functional and morphologic consequences of light exposure in primate eyes. Invest Ophthalmol Vis Sci 53:6035-6044.2012).

Organisciak DT, Vaughan DK (Retinal light damage: mechanisms and protection. Prog Retin Eye Res 29:113-134.2010).

Ortin-Martinez A, Valiente-Soriano FJ, Garcia-Ayuso D, Alarcon-Martinez L, JimenezLopez M, Bernal-Garro JM, Nieto-Lopez L, Nadal-Nicolas FM, Villegas-Perez MP, Wheeler LA, Vidal-Sanz M (A novel in vivo model of focal light emitting diodeinduced cone-photoreceptor phototoxicity: neuroprotection afforded by brimonidine, BDNF, PEDF or bFGF. PLoS One 9:e113798.2014).

Peng M-L, Tsai C-Y, Chien C-L, Hsiao JC-J, Huang S-Y, Lee C-J, Lin H-Y, Wen Y-C, Tseng K-W (The influence of low-powered family LED lighting on eyes in mice experimental model. Life Science J 9:477-482.2012).

Pinazo-Duran MD, Gallego-Pinazo R, Garcia-Medina JJ, Zanon-Moreno V, Nucci C, DolzMarco R, Martinez-Castillo S, Galbis-Estrada C, Marco-Ramirez C, Lopez-Galvez MI, Galarreta DJ, Diaz-Llopis M (Oxidative stress and its downstream signaling in aging eyes. Clin Interv Aging 9:637-652.2014).

Protection ICoN-IR (ICNIRP Guidelines on limits of exposure to incoherent visible and 
infrared radiation. Health Physics 105:74-96.2013).

Rizzolo LJ (Polarity and the development of the outer blood-retinal barrier. Histol Histopathol 12:1057-1067.1997).

Shang YM, Wang GS, Sliney D, Yang CH, Lee LL (White light-emitting diodes (LEDs) at domestic lighting levels and retinal injury in a rat model. Environ Health Perspect 122:269-276.2014).

Sliney $\mathrm{DH}$ (Exposure geometry and spectral environment determine photobiological effects on the human eye. Photochem Photobiol 81:483-489.2005).

Stone J, Maslim J, Valter-Kocsi K, Mervin K, Bowers F, Chu Y, Barnett N, Provis J, Lewis G, Fisher SK, Bisti S, Gargini C, Cervetto L, Merin S, Peer J (Mechanisms of photoreceptor death and survival in mammalian retina. Prog Retin Eye Res 18:689735.1999).

Sui GY, Liu GC, Liu GY, Gao YY, Deng Y, Wang WY, Tong SH, Wang L (Is sunlight exposure a risk factor for age-related macular degeneration? A systematic review and meta-analysis. Br J Ophthalmol 97:389-394.2013).

Ueda T, Nakanishi-Ueda T, Yasuhara H, Koide R, Dawson WW (Eye damage control by reduced blue illumination. Exp Eye Res 89:863-868.2009).

van Norren D, Gorgels TG (The action spectrum of photochemical damage to the retina: a review of monochromatic threshold data. Photochem Photobiol 87:747-753.2011).

Wenzel A, Grimm C, Samardzija M, Reme CE (Molecular mechanisms of light-induced photoreceptor apoptosis and neuroprotection for retinal degeneration. Prog Retin Eye Res 24:275-306.2005).

Youssef PN, Sheibani N, Albert DM (Retinal light toxicity. Eye (Lond) 25:1-14.2011). 
Captions to figures:

\section{Figure 1: LED device and exposure protocols.}

A: LED containing device: all the walls of the animal's compartment were equipped with LEDs. B: acute exposure protocol: rats were kept in the normal cyclic light of the animal facilities for 3 weeks. Before exposure to LEDs on the device seen on panel $A$, they were dark-adapted and their pupils were dilated with atropin before LED exposure $(6000,1500$, 1000 or $500 \mathrm{~lx}$ ). After $24 \mathrm{~h}$ of exposure they were returned to the animal facility for 7 days and then sacrificed. C: Long term exposure protocol: After the same stabulation period than before, the rats were exposed in the LED device, cyclically ( $12 \mathrm{~h}$ dark $12 \mathrm{~h}$ light, 500 Ix) for one week or 1 month and then sacrificed.

Figure 2: Retinal degeneration induced in albino Wistar and pigmented Long Evans rats by a single exposure to $\mathbf{6 0 0 0}$ lux for $\mathbf{2 4 h}$ : Wistar or Long Evans Rats $(N=4)$ were exposed for $24 \mathrm{~h}$ to 6000 lux white light. One week after the exposure the animals were sacrificed as described on figure 1B. Afterwards, the eyes were fixed, included in paraffin, sectioned and stained with hematoxiline-eosine (lower part of both panels). The photoreceptors' nuclei were counted in the superior and inferior retina. The light was obtained using either a LED, a CCFL or CFL source. A: Long Evans Rats, B: Wistar rats. ${ }^{*}: p<0.05,{ }^{* *}: p<0.01,{ }^{* * *}: p<0.001$, SEM were used for graph and SD for statistical work. Significance was evaluated using the Conover-Inan statistical test). In lower images the scale bar represents $50 \mu \mathrm{m}$.

Figure 3: Modifications induced in GFAP expression, rods (Rho4D2), cones (PNA) and inflammatory cells (IBA1 and ED1) by white LED exposure: Wistar or Long Evans Rats $(\mathrm{N}=4)$ were exposed for $24 \mathrm{~h}$ to white LED light (6000 lux). One week after the exposure, the animals were sacrificed as described on figure 1B. Afterwards, the eyes were fixed, included in OCT, cryo-sectioned and immunolabelled using different antibodies. NE: retinas from non-exposed animals used as control, LED: retinas from rats exposed to white LEDs. DAPI-GFAP row shows GFAP labeling in green counterstained with DAPI in blue. Insets show details of the Müller cells expansions. Rho4D2 row shows labeling of rods, PNA row labeling of cones. The lower row was labeled with anti-lba1 in green and anti-ED1 in red, unveiling macrophages and microglia. Cells labeled with both antibodies are seen in yellow. A DAPI counterstained is shown in blue. Scale bar 
represents $20 \mu \mathrm{m}$.

Figure 4: Modifications induced in GFAP expression, rods, cones and inflammatory cells by exposure to CCFL or CFL: Wistar (W) or Long Evans (LE) Rats ( $N=4)$ were exposed for $24 \mathrm{~h}$ to CCFL or CFL light (6000 lux). One week after the exposure the animals were sacrificed as described on figure 1B. Afterwards, the eyes were fixed, included in OCT, cryo-sectioned and immunolabelled using different antibodies. Control images for these labeling can be seen on figure 3. DAPI-GFAP row shows GFAP labeling in green counterstained with DAPI in blue. Rho4D2 row shows labeling of rods, PNA row labeling of cones. The lower row was labelled with anti-lba1 in green and anti-ED1 in red, unveiling macrophages and microglia. Cells labeled with both antibodies are seen in yellow. Insets show details of this double labeling. A DAPI counterstained is shown in blue. Scale bar represents $20 \mu \mathrm{m}$.

Figure 5: Retinal degeneration induced in albino Wistar and pigmented Long Evans rats by a single exposure to different luminance of white LED and CFL during 24h : Wistar or Long Evans Rats $(\mathrm{N}=4)$ were exposed for $24 \mathrm{~h}$ to different luminance of white LED or to CFL $500 \mathrm{~lx}$. One week after the exposure the animals were sacrificed as described on figure 1B. Afterwards, the eyes were fixed, included in paraffin, sectioned and stained with hematoxiline-eosin (lower part of both panels). The photoreceptors' nuclei were counted in the superior and inferior retina. The light was obtained using either a LED source or a CFL source. A: Long Evans Rats, B: Wistar rats. $\left({ }^{*}: p<0.05,{ }^{* *}: p<0.01,{ }^{* * *}\right.$ : $p<0.001$, SEM were used for graph and SD for statistical work, Significance was evaluated using the Conover-inan statistical test).

Figure 6: Modifications induced in GFAP expression, rods and cones by exposure to different luminances of white LED (500 and 1500 lx): Wistar (W) or Long Evans (LE) Rats ( $N=4)$ were exposed for $24 \mathrm{~h}$ to white LED (500 and $1500 \mathrm{~lx}$ ). One week after the exposure the animals were sacrificed as described on figure 1B. Afterwards, the eyes were fixed, included in OCT, cryo-sectioned and immunolabeled using different antibodies. Control images for these labeling can be seen on figure 3. DAPI-GFAP row shows GFAP labeling in green counterstained with DAPI in blue. Rho4D2 row shows labeling of rods, PNA row labeling of cones, Insets show details of the Müller cells expansions. Scale bar represents $20 \mu \mathrm{m}$. 
Figure 7: Leakage of the outer retinal barrier. Wistar or Long Evans Rats $(\mathrm{N}=4)$ were exposed for $24 \mathrm{~h}$ to white LEDs at $1500 \mathrm{~lx}$. One week after the exposure the animals were sacrificed as described on figure 1B. Afterwards, the eyes were fixed, included in OCT, cryo-sectioned and immunolabeled using anti rat serum albumin. NE: non-exposed control rats. LED: LED-exposed rats. White arrows indicate the regions of leakage. Scale bar represents $25 \mu \mathrm{m}$.

Figure 8: Retinal degeneration induced in albino Wistar rats by a cyclic exposure (15h light/12h dark) to 500 Ix of white, blue or green LEDs: Wistar rats $(\mathrm{N}=4)$ were exposed cyclically to 500 Ix of LED light for 1 week or 1 month. After the exposure the animals were sacrificed as described on figure 1C. Afterwards, the eyes were fixed, included in paraffin, sectioned and stained with hematoxiline-eosin. The photoreceptors' nuclei were counted in the superior and inferior retina. A: 1 week of exposure, $B: 1$ month of exposure. $\left({ }^{*}: p<0.05,{ }^{* *}: p<0.01,{ }^{* *}: p<0.001\right.$, SEM were used for graph and SD for statistical work. Significance was evaluated using the Conover-Inan statistical test).

Figure 9: Retinal degeneration induced in pigmented Long Evans rats by a cyclic exposure (15h light/12h dark) to 500 Ix of white, blue or green LED or to fluocompact light: Long Evans rats $(\mathrm{N}=4)$ were exposed cyclically to 500 Ix of LED or fluocompact light for 1 week or 1 month. After the exposure the animals were sacrificed as described on figure 1C. Afterwards, the eyes were fixed, included in paraffin, sectioned and stained with hematoxiline-eosin. The photoreceptors' nuclei were counted in the superior and inferior retina. A: 1 week of exposure, B: 1 month of exposure. $\left(^{*}: p<0.05,{ }^{* *}: p<0.01,{ }^{* * *}\right.$ : $p<0.001$, SEM were used for graph and SD for statistical work. Significance was evaluated using the Conover-Inan statistical test).

Figure 10: Modifications induced in GFAP expression, rods and cones by the exposure of a cyclic with LED (500 LX) for one month. Wistar or Long Evans Rats $(\mathrm{N}=4)$ were exposed cyclically to 500 Ix of white, green or blue LED light for 1 month. After the exposure, the animals were sacrificed as described on figure $1 \mathrm{C}$. Afterwards, the eyes were fixed, included in OCT, cryo-sectioned and immunolabeled using different antibodies. Control images for these labeling can be seen on figure 3. GFAP row shows GFAP labeling in green. Rho4D2 row shows labeling of rods, PNA row shows labeling of cones. 
Scale bar represents $35 \mu \mathrm{m}$.

Figure 11: Modifications induced in scotopic full-field electroretinogram by the exposure of a cyclic with LED (500 Ix) for one month. The scotopic amplitudes of aand b-waves and implicit times of the same ERG waves were represented as the delta (variation) between exposed and non-exposed animals. Scotopic ERG were recorded before and after illumination. Following an overnight dark-adaptation, animals were anaesthetized and their pupils dilated; stimuli consisted of light flashes of 9 increasing intensities delivered through a Ganzfeld bowl $\left(0.0003\right.$ cd.s. $\mathrm{m}^{2}$ to 10 cd.s. $\mathrm{m}^{2}$ here expressed in log scale intensities, Siem Biomedicale). Responses to 5 flashs per intensity were averaged (Visiosystem software). Mean variation of the a- (left column) and b-waves (right column) amplitudes (first row) and implicit times (second row) were compared between albino Wistar (W, white dots) and pigmented (LE, black dots) rats. As the a-wave is a negative wave, a positive variation is an alteration of the a-wave amplitude. As the bwave is a positive wave, a negative variation is an alteration of the b-wave amplitude. Here, both scotopic a- and b- waves' amplitudes are impaired by white LED illumination, in albino $(\mathrm{W})$ as well as in pigmented (LE) rats. Significance of the difference between $\mathrm{W}$ and LE variation of the a- and b-waves amplitudes was evaluated using the Mann-Whitney non-parametric test. Asterix indicate significant differences ${ }^{*} p<0.05,{ }^{* *} p<0.005$. The aand b-waves implicit times were not significantly affected by this illumination protocol. 
A
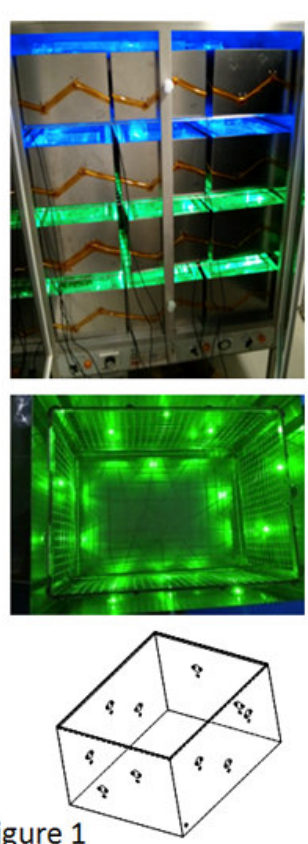

B

Acute exposure protocol

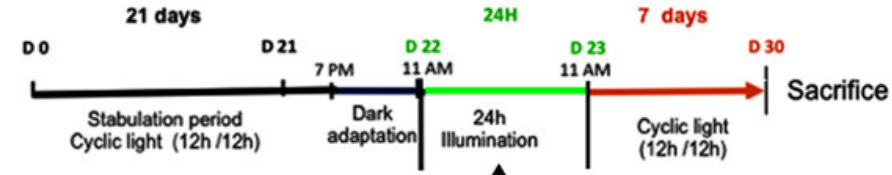
Atropine

LED: 6000 to $500 \mathrm{~lx}$

CCFL: $6000 \mathrm{~lx}$

CFL: $6000+500 \mathrm{~lx}$

C

Long-term exposure protocole

Do

D21

D29

D 43

Stabulation period
Cyclic light (12h/12h)

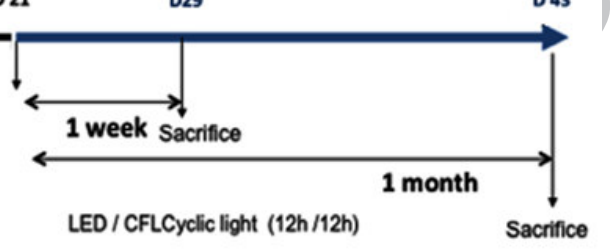

Figure 1 
A
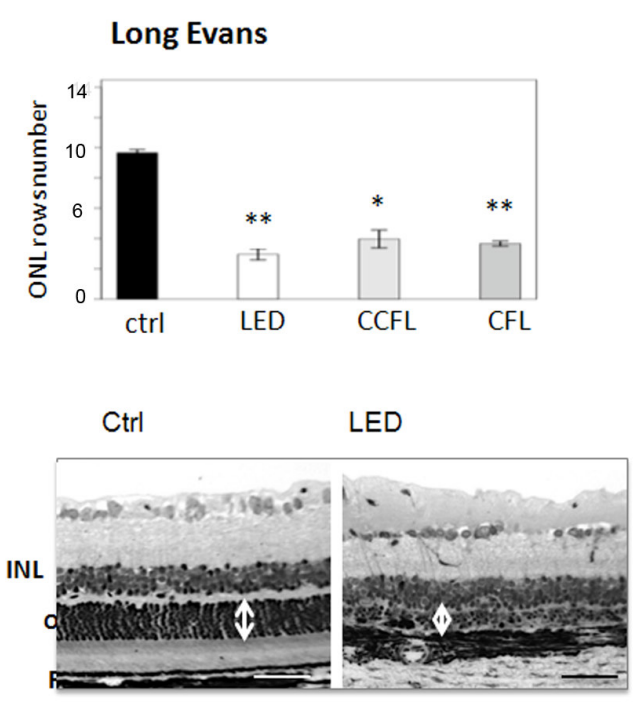

B
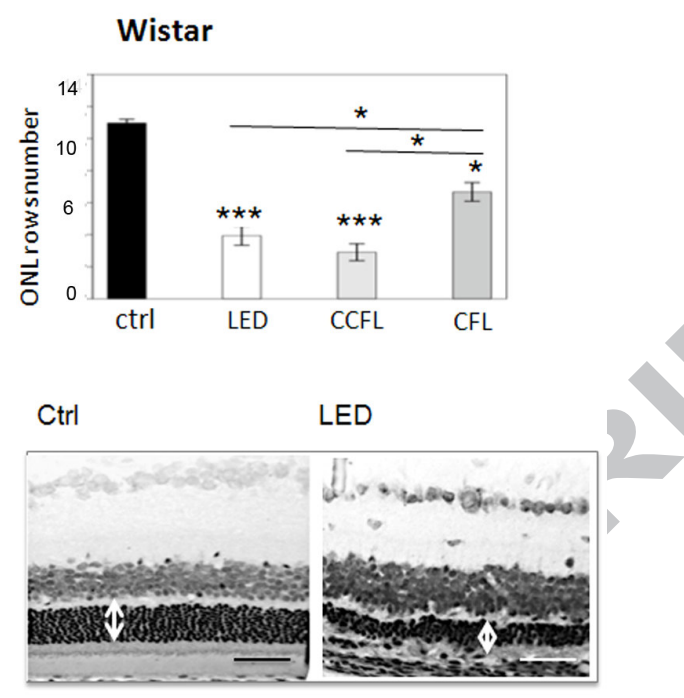
LE

NE
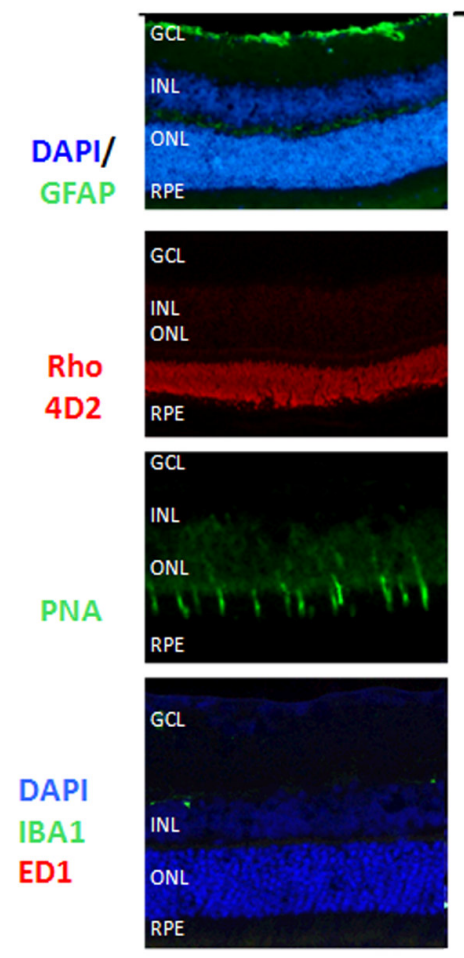

LED
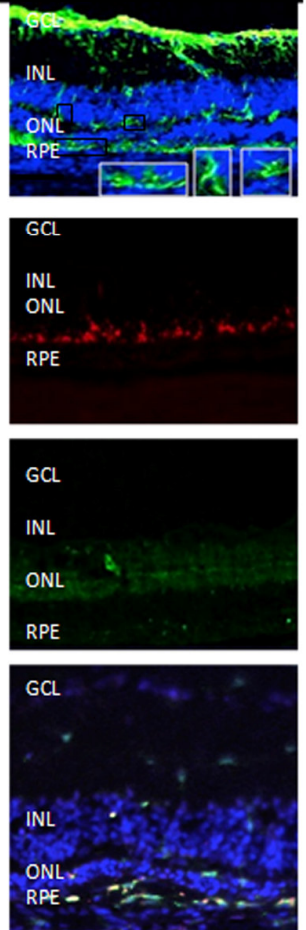

Wistar

NE
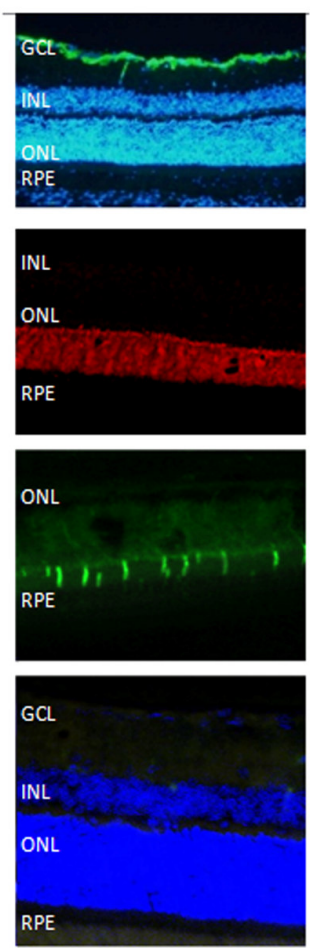

LED
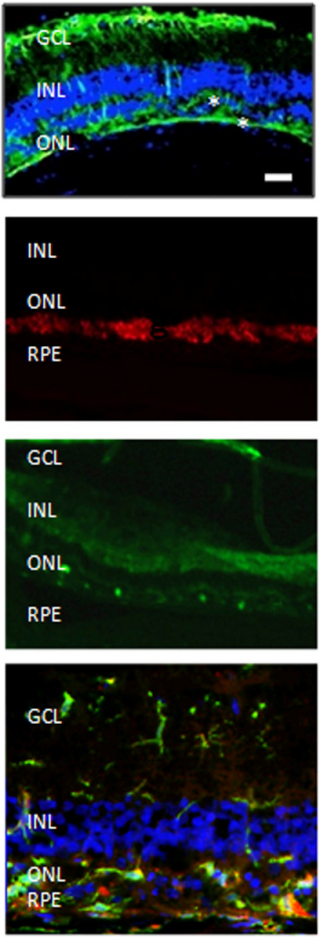


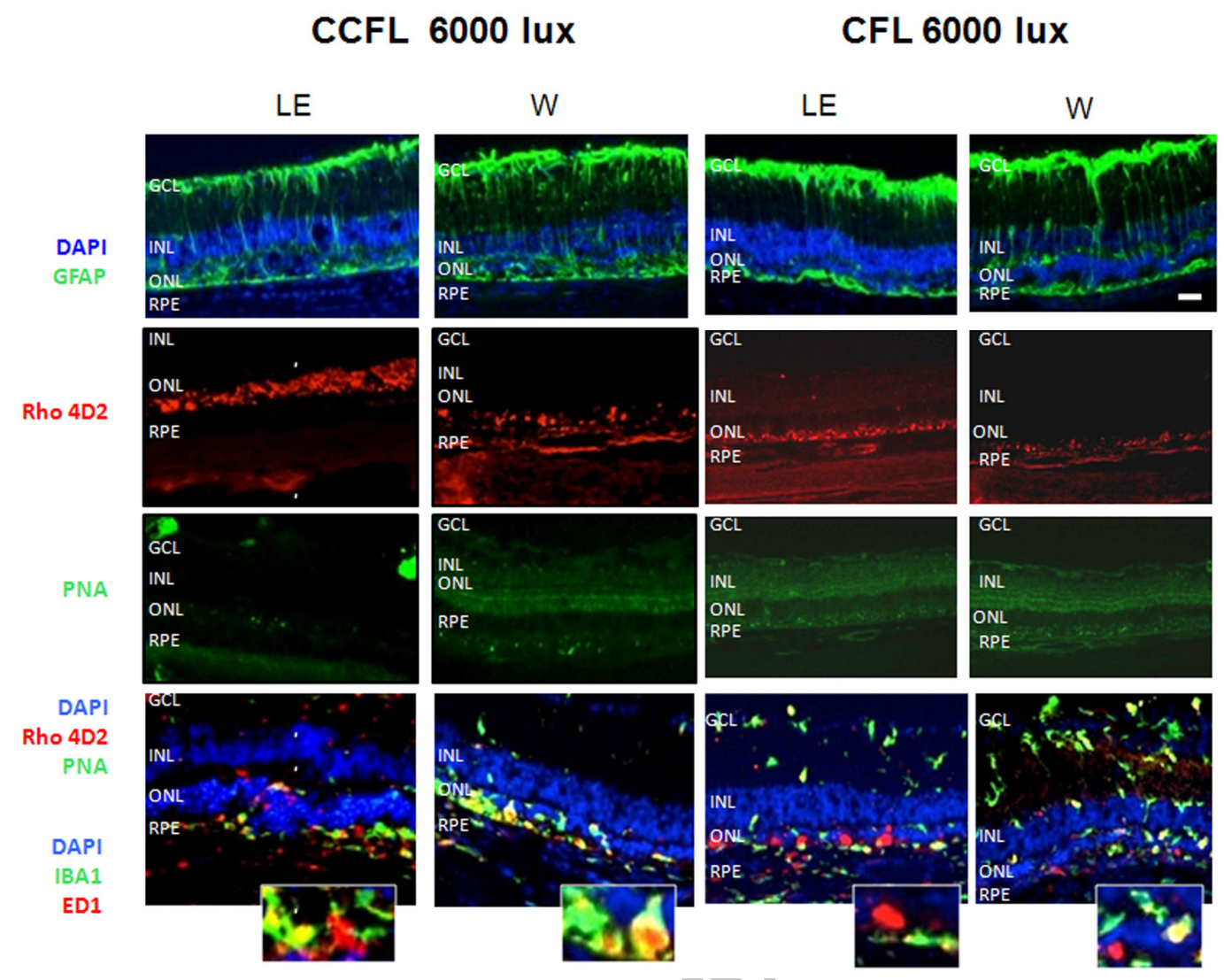


A

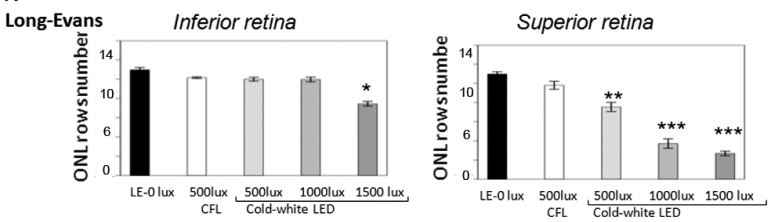

B

Wistar

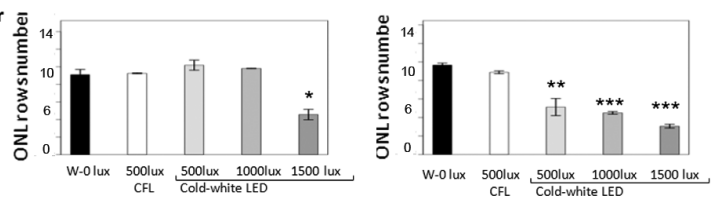




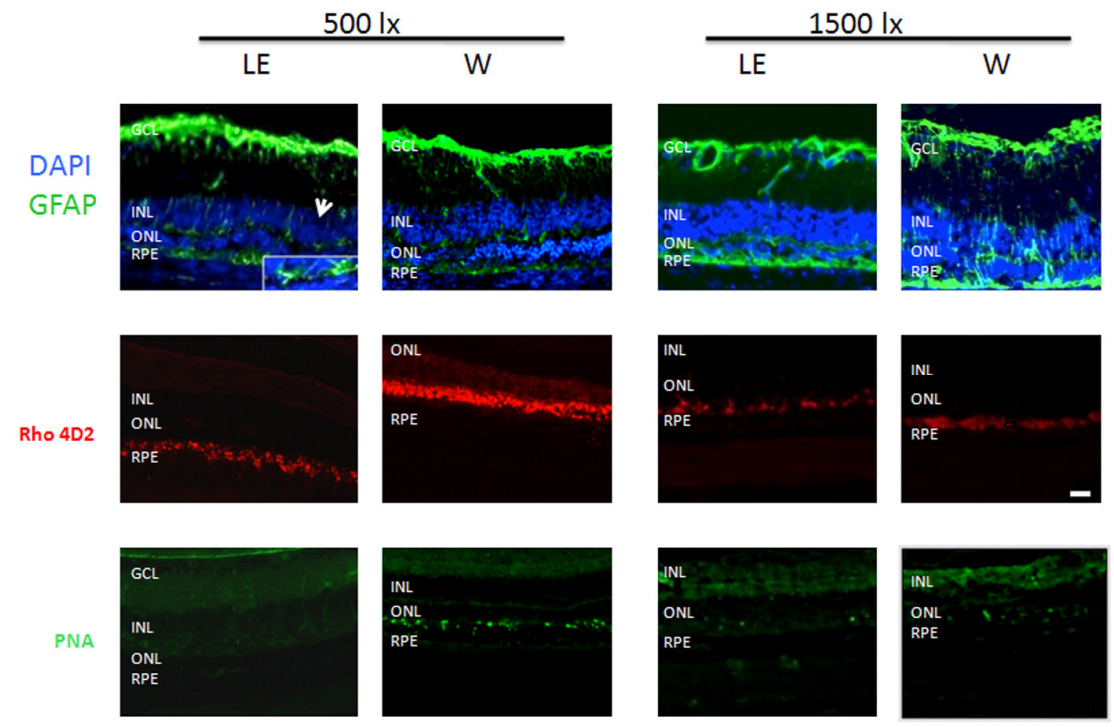




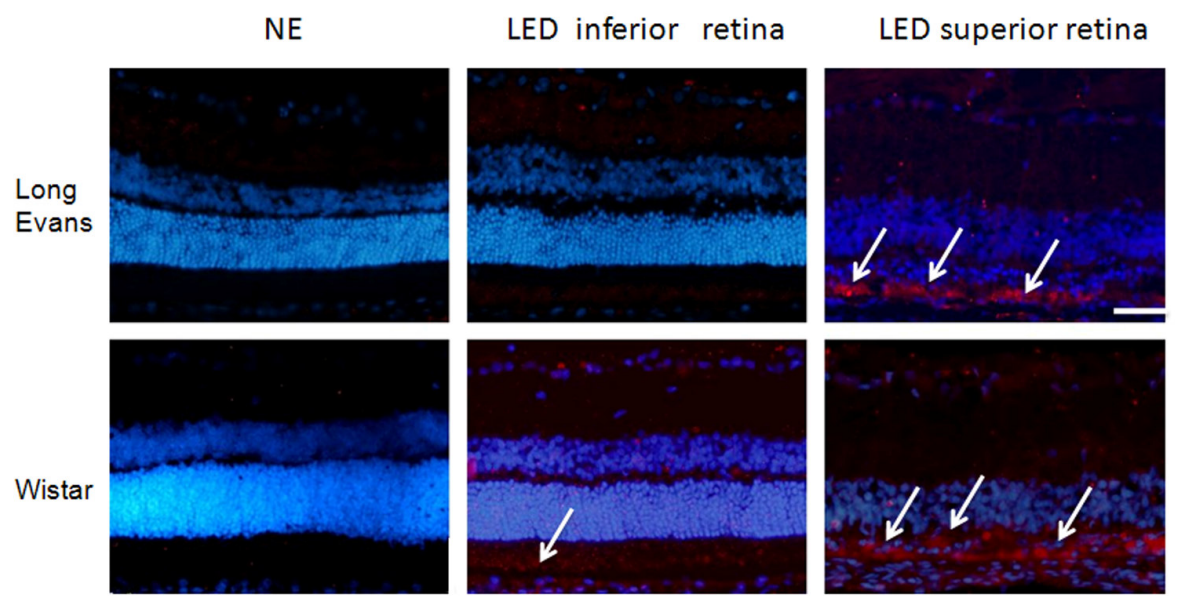

DAPI Rat albumin 
A 1 week Inferior

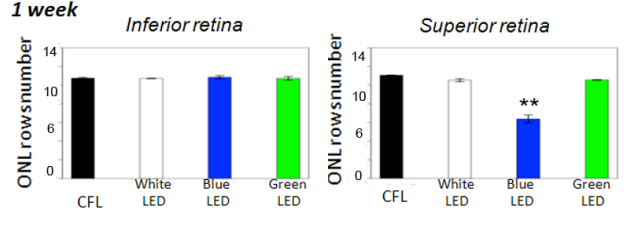

WISTAR

B
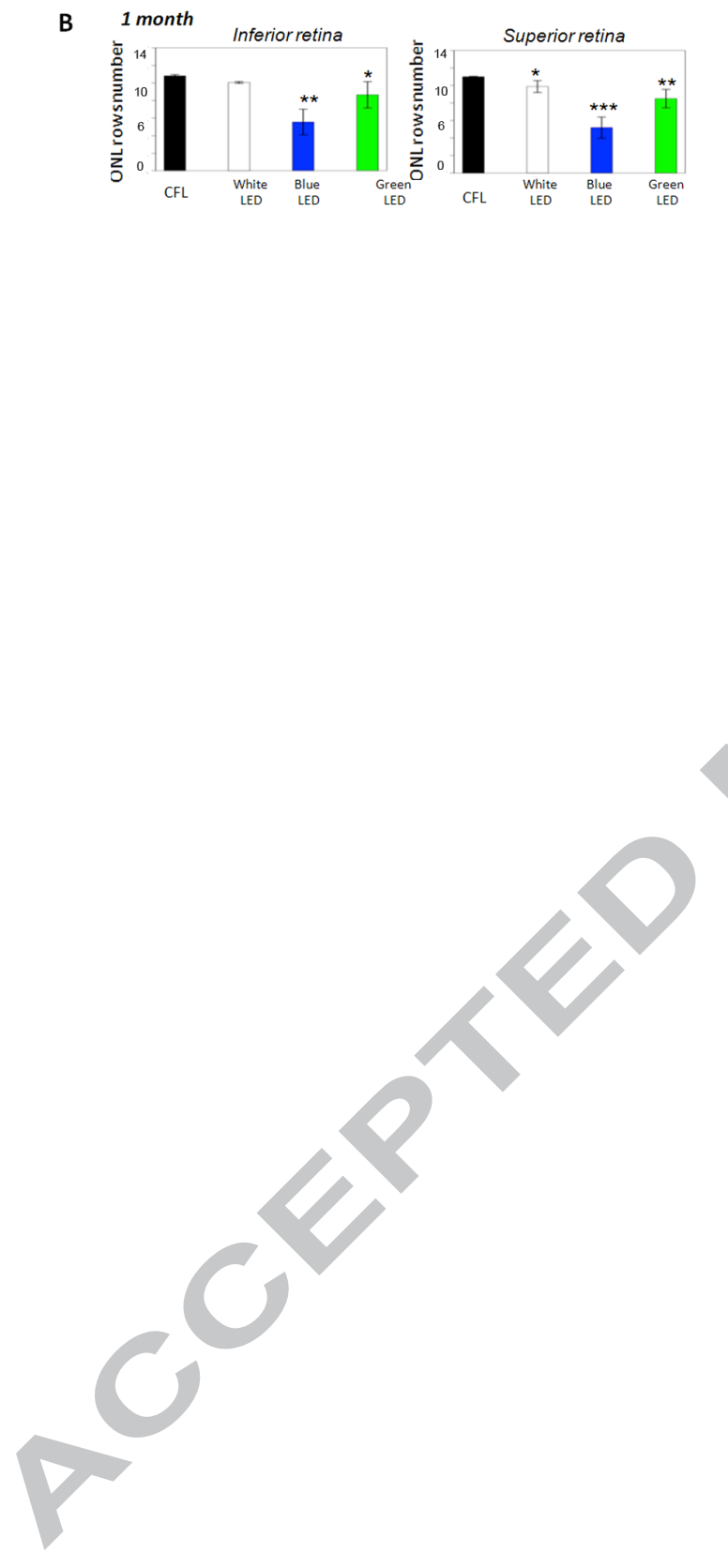
A

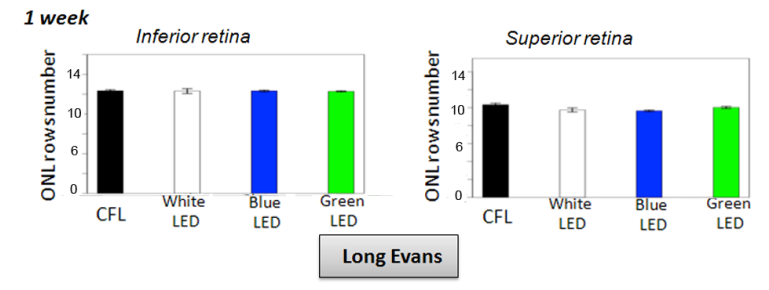

B 1 month

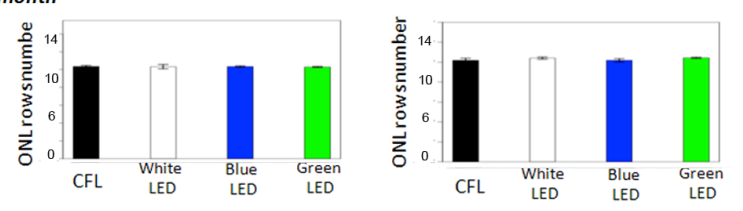




\section{ACCEPTED MANUSCRIPT}

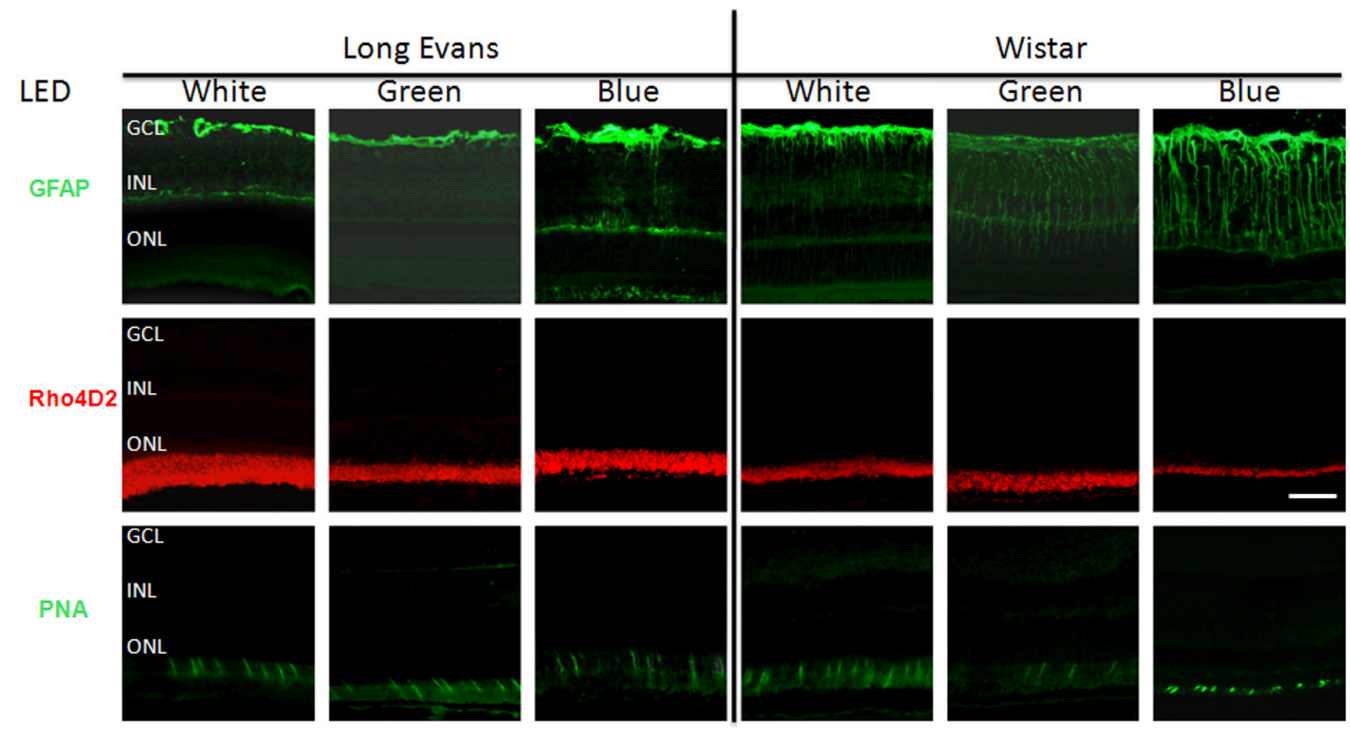


Variation of the Scotopic a wave amplitude Variation of the Scotopic b wave amplitude
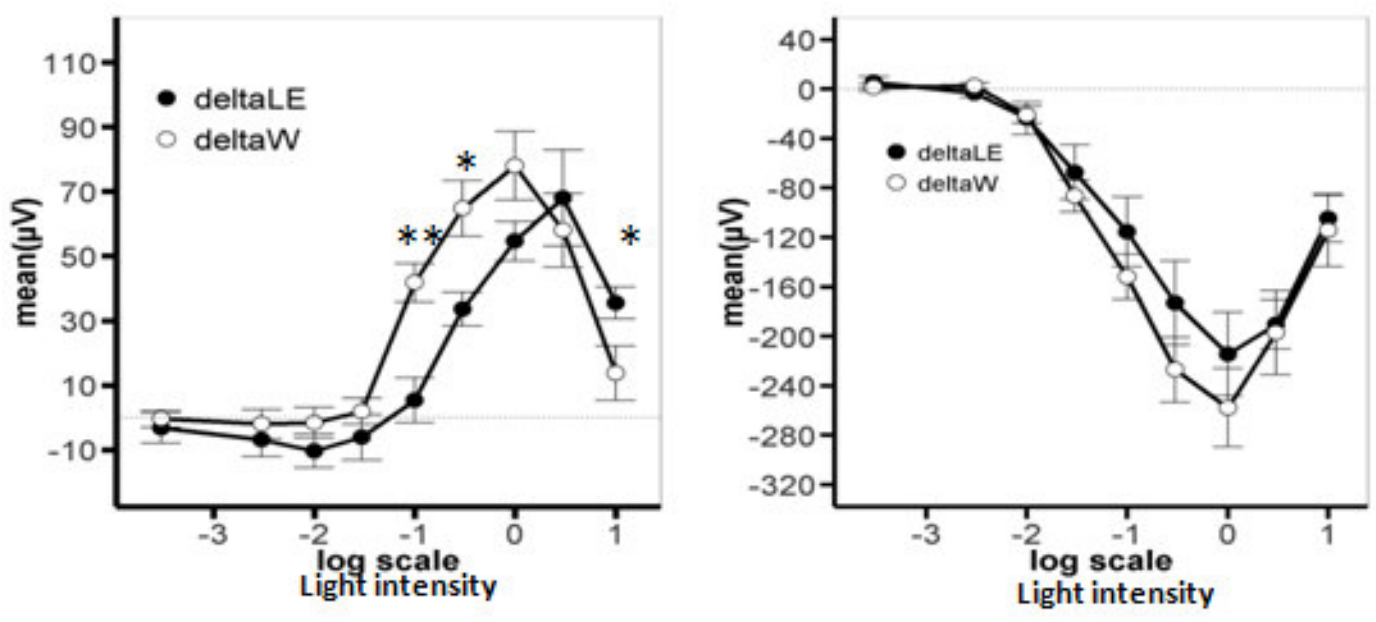

Variation of the Scotopic a wave implicit time

Variation of the Scotopic

B wave implicit time
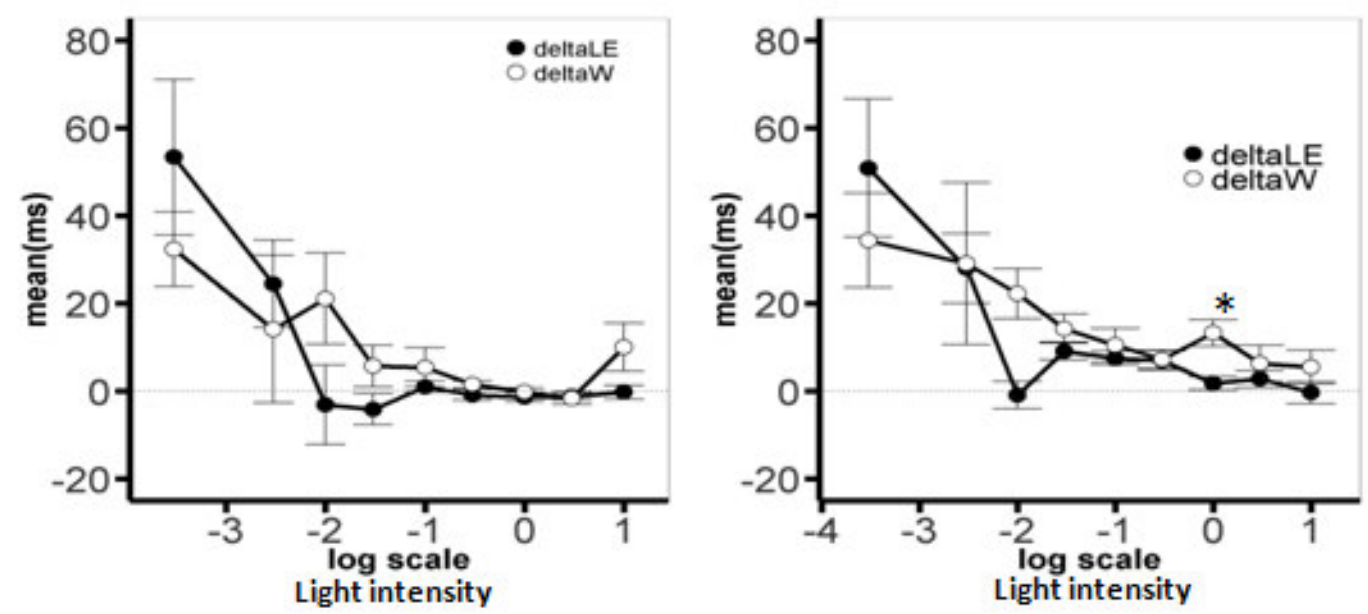


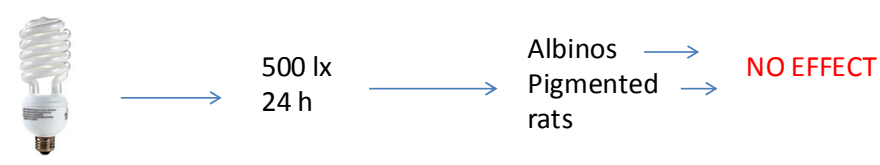

FLC

$\prod \longrightarrow \begin{array}{ll}500 \mathrm{~lx} \\ 24 \mathrm{~h} \longrightarrow\end{array}$

LED

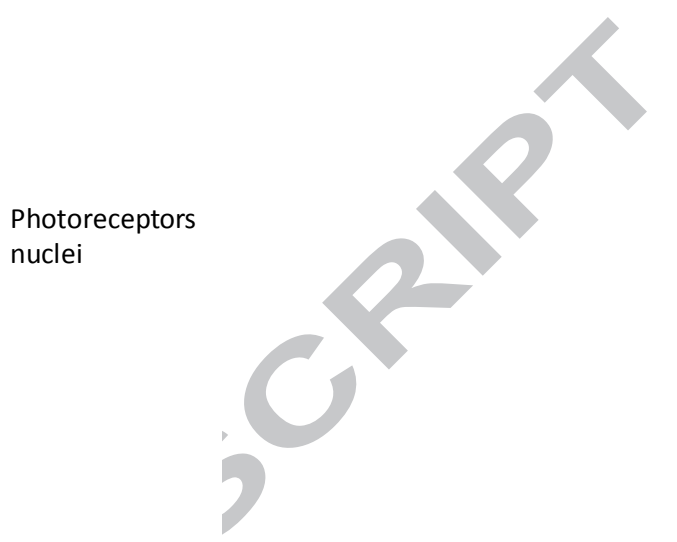

